\title{
Risk of Foot Ulcer Development in Diabetic Patients - Relation to Isokinetic Muscle Strength, Sensory Function, and Clinical Findings
}

Anne Sofie Bruno Pedersen ${ }^{1}$ Laerke Walther Junggreen Have ${ }^{1}$ Henning Andersen ${ }^{1,2}$ Niels Ejskjaer ${ }^{1}$ and Christer Zoeylner Swan ${ }^{1,2,3 *}$

${ }^{1}$ Clinical Neurology Research Group, Department of Neurology and Endocrinology, Aarhus University Hospital, Palle Juul-Jensen Boulevard 165, 8200 Aarhus N., Denmark

${ }^{2}$ Department of Clinical Medicine, Aarhus University, Palle Juul-Jensens Boulevard 82, 8200 Aarhus N., Denmark

${ }^{3}$ Department of Otorhinolaryngology and Head \& Neck Surgery, Aarhus University Hospital, Palle Juul-Jensen Boulevard 165, 8200 Aarhus N., Denmark

${ }^{*}$ Corresponding author: Christer Zoeylner Swan, Department of Otorhinolaryngology and Head \& Neck Surgery, Aarhus University Hospital, Palle Juul-Jensen Boulevard 165, 8200 Aarhus N., Denmark; Tel: +45 20259181; Email: csa@clin.au.dk

Received: April 05, 2019; Accepted: April 17, 2019; Published: May 01, 2019;

\begin{abstract}
Aim: To investigate whether reduced muscle strength in the lower extremities in diabetic patients is associated to the development of Diabetic Foot Ulcer (DFU).

Methods: We conducted a retrospective cohort study on 95 diabetic patients who participated in studies on Diabetic Polyneuropathy (DPN) and motor function 12-16 years earlier. Isokinetic muscle strength at the ankle and knee, Neurological Impairment Scores (NIS), vibration perception thresholds (VPT), and demographic data were obtained from the initial studies. Patient files were systematically reviewed, and information on DFU occurrence and Macrovascular Disease (MVD) acquired.
\end{abstract}

Results: Twenty-six patients developed DFU. A temporal relationship was found for development of DFU among patients with reduced strength at both the ankle and knee (all $\mathrm{P}<0.05)$. Univariate analyses showed a relationship between DFU and reduced strength for ankle dorsal flexion ( $<<0.001)$, ankle plantar flexion $(\mathrm{P}<0.005)$, knee extension $(\mathrm{P}<0.001)$, and knee flexion $(\mathrm{P}<0.005)$. DFU was related to NIS $(\mathrm{P}<0.001)$ and MVD $(\mathrm{P}<0.05)$ in both univariate and multivariate regression analyses. After adjustment for MVD, all strength measures were related to DFU. When adjusting for NIS, a trend was only found for ankle dorsal flexion $(\mathrm{P}=0.08)$.

Conclusions: In DPN, muscle weakness at the ankle and knee contributes to development of foot ulcers.

Keywords: Type 1 diabetes mellitus, type 2 diabetes mellitus, polyneuropathy, muscle strength, foot ulcer, follow-up.

\section{Introduction}

Diabetic Foot Ulcers (DFU) lead to reduced quality of life in affected patients and impose considerable health care costs [1,2]. Several risk factors have been linked to the occurrence of ulceration in both type 1 and type 2 diabetic patients, including impaired glycaemic control, peripheral arterial disease, and Diabetic Polyneuropathy (DPN) [3-6]. Reduced sensation due to DPN allows minor trauma to evolve undetected, thereby contributing to the initial stages of foot ulceration [7]. In recent years also motor dysfunction as a result of DPN, has been implicated in the pathology underlying DFU. DPN leads to muscle atrophy and loss of muscle strength in the foot and in more advanced cases also in the lower legs [8-11]. In addition, patients with DPN develop foot deformities such as bony prominences and metatarsophalangeal joint deformities, which contribute to abnormalities of pressure distribution and ulcer formation [5, 12-14]. Exposure of pathologically altered bony structures due to atrophy of overlying tissue, concomitant with increased forefoot slap during gait cycle deceleration as a result of reduced muscle strength for ankle dorsal flexion, is believed to cause increased plantar pressure during gait, which is associated to foot ulceration [15-17]. Weakness of dorsal flexion at the ankle and great toe combined with reflex testing, have been identified as risk factors for development of DFU [18]. However, as muscle strength was assessed by manual testing, the degree of muscle weakness was likely underestimated which may have weakened the association to DFU development [19]. Isokinetic dynamometry provides a more accurate quantification of muscle strength in neuropathic patients which could strengthen the proposed relationship between loss of muscle strength in the lower extremities due to DPN and foot ulcer formation [20].

In the present retrospective study, we have evaluated the temporal relationship between reduced muscle strength at both the knee and ankle determined by isokinetic dynamometry and the occurrence of DFU. We included diabetic patients evaluated using isokinetic 
dynamometry 12-16 years previously. The patients were followed up by obtaining data collected from patient files with focus on the occurrence of DFU.

\section{Materials and Methods}

\section{Study design}

We conducted a retrospective cohort-study on 95 diabetic patients (65 type 1 and 30 type 2 ) who were recruited for cross-sectional studies on diabetic polyneuropathy and muscle strength at our laboratory 12 16 years prior to the present study [9,21]. Baseline data on isokinetic muscle strength at the ankle and knee, age, height, weight, Neuropathy Impairment Score (NIS), and Vibratory Perception Threshold (VPT) were acquired from the initial study protocol. Patient hospital files were then systematically reviewed for the period since participation in the initial studies until $31^{\text {st }}$ of December 2009, and information on foot ulcer occurrence and Macrovascular Disease (MVD) (defined as claudication, acute myocardial infarction, transient ischemic attack, or stroke) were recorded. A DFU was defined as an ulcer that required specialized treatment at the Diabetic Foot Centre located at the Department of Endocrinology, Aarhus University Hospital.

The study was approved by the Danish Data Protection Agency (Journal No. 2010-41-4811).

\section{Measurements performed in initial cross-sectional studies}

In the initial studies by Andersen et al. isokinetic dynamometry (Lido Active Multijoint; Loredan Biomedical, West Sacramento, CA) was applied to measure maximal muscle strength for extension and flexion of the knee and dorsal and plantar flexion of the ankle [9,21]. Maximal strength was measured as peak torque at slow movement velocities with subjects in a sitting position. Straps were applied proximally and distally to the respective joints. For ankle measurements, the foot was secured to a footplate. Standardized verbal feedback was given during the procedures.

NIS is a score obtained by performing a standardized clinical evaluation of muscle strength, activity of tendon reflexes, and sensation at the great toe and index finger. In the present study, all points due to muscle weakness have been omitted to avoid correlation bias.

VPT was evaluated at the dominant index finger pulp and nondominant dorsum of the great toe, as described by Dyck et al [21].

\section{Study population}

Baseline data were obtained for 115 patients. Twenty patients were excluded as eleven patient files could not be located from the outpatient clinic archives, two patients had moved out of the geographical area, and five patients were lost to follow-up as they did not attend scheduled appointments at the outpatient clinic. Finally, foot ulceration following penetrating trauma complicated by infection occurred in one patient, and one patient had received chemotherapy for non-Hodgkin lymphoma known to cause polyneuropathy as a possible side effect. Thus, 95 patients were included in the present follow-up study.

In the initial studies, inclusion criteria for type 1 diabetic patients were duration of diabetes $\geq 20$ years and age $<65$ years, and for type 2 diabetic patients diabetes duration $\geq 5$ years and age $<70$ years. In short the exclusion criteria applied, secured that no participant had reduced levels of activity due to cardiac or lung disease, musculoskeletal disorders, suffered any neurological disorder or other endocrine disorder than diabetes mellitus, or was subject to symptomatic macroangiopathy. The criteria are stated in detail in the initial studies by Andersen et al $[9,21]$.

\section{Statistical analysis}

Absolute values for muscle strength were converted to percentage of expected strength based on calculations adjusting for age, body mass, height, and gender as described earlier [21].

The two-sample t-test and Pearson's Chi2 test were applied to compare demographic data in the group of patients that developed DFU with the group without DFU.

Logistic regression analysis was applied to evaluate the relationship between ulcer occurrence and degree of muscle weakness. Multiple logistic regression analysis was applied when adjusting for MVD and NIS, and to evaluate the influence of the strength measurements combined. As a minimum of ten outcome events are needed per predictor variable when performing multivariate analysis, only two variables were included in each multivariate analysis model [23].

For each strength measurement, patients were divided in groups according to percent of expected muscle strength; group 1: 0-74\%, group 2: $75-99 \%$ and group $3: \geq 100 \%$. The groups were then compared using the log-rank test in order to evaluate the temporal relationship between muscle strength and DFU.

\section{Results}

The follow-up period was 13.9 (0.2-15.9) [median (range)] years, during which 26 patients developed DFU. No difference was found for diabetes type (65\% type 1 vs. $70 \%$ type 1$)$, age [54 [(26-69) years vs. 47 (26-69) years], duration of diabetes [26 (8-48) years vs. 23 (141) years], BMI [25 (19-37) vs. 25 (17-40)], or gender composition ( $19 \%$ female vs. $36 \%$ female) between patients who developed DFU and those without DFU, respectively (all P $>0.05$ ). More patients with DFU were diagnosed with MVD during follow-up, as compared to patients that did not develop DFU ( $54 \%$ vs. $19 \%, \mathrm{P}<0.005$ ).

Muscle strength, expressed as percent of expected strength, was lower at baseline for patients that developed DFU during follow-up than for patients that did not develop DFU for both ankle dorsal [68 $(25-104) \%$ vs. $92(49-126) \%, \mathrm{P}<0.0001]$ and plantar $[73(40-100) \%$ vs. $85(42-145) \%, \mathrm{P}<0.01$ ] flexion, as well as knee flexion [76 (50$103) \%$ vs. 90 (56-129)\%, $\mathrm{P}<0.01]$ and extension $[74(55-106) \%$ vs. 89 (59-133)\%, P < 0.0001]. Further, NIS [24 (3-38) vs. 6 (0-31), P $<0.0001$ ] and VPT [99 (11-99) vs. 95 (32-99), P <0.01] scores were higher for patients who developed DFU.

Univariate analysis established a correlation between reduced muscle strength for both dorsal and plantar flexion at the ankle and flexion and extension at the knee, and the occurrence of DFU (Table 1). The odds ratios express the risk of developing DFU if muscle strength is reduced by one percent. Also, a high NIS and the occurrence of 
MVD were associated to foot ulceration. VPT, however, showed no relationship to DFU development.

Results of the multivariate regression analyses are shown in table 2. Reduced muscle strength for ankle dorsal flexion, ankle plantar flexion, knee extension, and knee flexion were all related to DFU occurrence when adjusting for MVD as shown in model 1, a-d. In model 2, muscle strength for flexion and extension at the ankle and knee were combined with NIS. All analyses found NIS to be an independent risk factor for DFU development, whereas a tendency was found for dorsal flexion, only. For movements at the ankle and knee, only ankle dorsal flexion and knee extension were related to DFU development (model
3 , $a+b)$. When combining ankle dorsal flexion and knee extension, only ankle dorsal flexion showed a relationship to DFU formation (model $3, c$ ). NIS and the occurrence of MVD are included in model 4 , and both were independently correlated to foot ulceration.

Figure 1, a-d, illustrates the association between lower extremity muscle strength and DFU development for patients grouped according to percentage of expected muscle strength at inclusion. For all movements measured, patients with expected strength of less than $75 \%$ had higher probabilities of developing DFU during follow-up. Patient groups did not differ with regard to distribution of diabetes type.

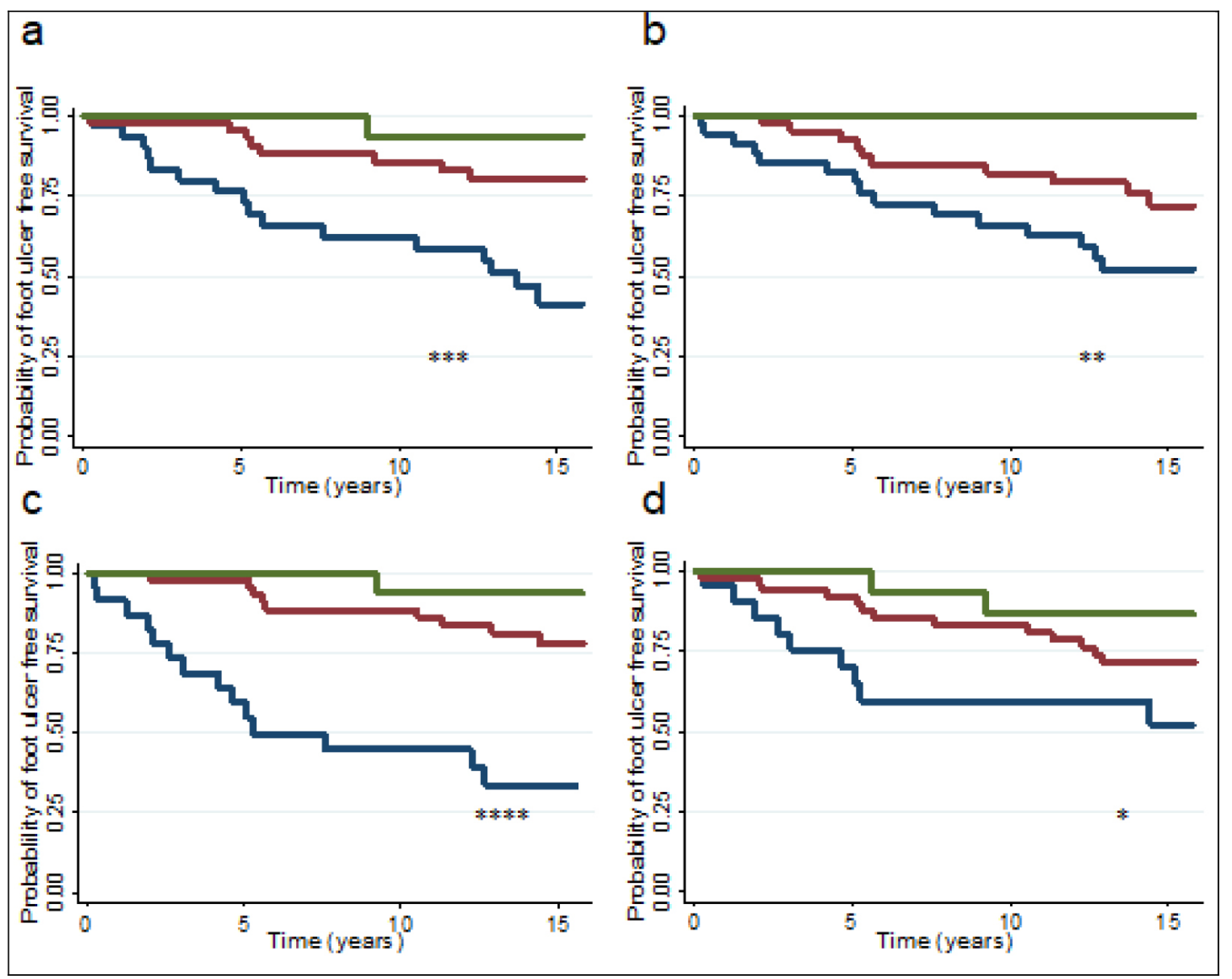

Figure 1. Kaplan Meier plot illustrating the probability of not developing foot ulcer in diabetic patients (65 type 1 and 30 type 2 ) according to percentage of expected strength for dorsal (a) and plantar (b) flexion at the ankle, and extension (c) and flexion (d) at the knee. Green line; patients with $>/=100 \%$ of expected strength for movement, red line; patients with $75-99 \%$ of expected strength for movement, and blue line; patients with $\leq 74 \%$ of expected strength for movement. $* \mathrm{P}<0.05,{ }^{*} \mathrm{P}<0.005,{ }^{*} * \mathrm{P}<0.0005$, and $* * * * \mathrm{P}<0.0001$

\section{Discussion}

In this retrospective cohort study, we investigated the relationship between muscle strength in the lower extremities and the risk of developing DFU in 95 diabetic patients. We found that reduced muscle strength in the lower extremities is a risk factor for ulceration of the foot in diabetes. Reduced strength for ankle dorsiflexion and knee extension proved to be the strongest risk factors for the development of DFU. The odds ratios calculated for the models shown in tables
1 and 2, express the risk of developing a foot ulcer during a 14-year period if muscle strength is reduced by $1 \%$. When applied on the results presented in Table 1, the finding is exemplified by the 10 fold increased risk of developing a foot ulcer found for a patient with an expected muscle strength for ankle dorsal flexion of $60 \%$ compared to that of a patient with normal muscle strength for this movement $(60 \%$ vs $\left.100 \% ; 1.06^{40}=10.3\right)$. 
Table 1. Odds ratios (OR) for univariate analysis expressing the risk of developing diabetic foot ulcers (DFU) in diabetic patients (65 type 1 and 30 type 2) in relation to muscle strength for movements at the ankle and knee, Neuropathy Impairment Score (NIS), and Vibratory Perception Threshold (VPT) at baseline, and macrovascular disease (MVD) developed during 13.9 (0.2-15.9) [median (range)] years follow-up. ORs for ankle and knee movements express the increased risk of developing DFU following a $1 \%$ reduction in muscle strength. All data are shown as OR and $95 \%$ confidence intervals $(95 \% \mathrm{CI}) . \mathrm{NS}=$ non-significant.

\begin{tabular}{|l|c|c|}
\hline & OR (95\% CI) & P \\
\hline Baseline & & $<0.001$ \\
\hline Ankle dorsal flexion & $1.06(1.02-1.09)$ & $<0.005$ \\
\hline Ankle plantar flexion & $1.04(1.01-1.07)$ & $<0.001$ \\
\hline Knee extension & $1.09(1.04-1.14)$ & $<0.005$ \\
\hline Knee flexion & $1.05(1.02-1.09)$ & $<0.001$ \\
\hline NIS & $1.15(1.09-1.22)$ & NS \\
\hline VPT & $1.03(0.98-1.08)$ & \\
\hline Consecutively recorded data & \\
\hline MVD & $5.03(1.89-13.4)$ & $<0.005$ \\
\hline
\end{tabular}

Table 2. Multivariate analyses in which two predictor variables are applied in each analysis in relation to diabetic foot ulcer occurrence during $13.9(0.2-15.9)$ [median (range)] years follow-up. In model 1, muscle strength for flexion and extension at the ankle and knee and macro vascular disease (MVD) are included. In model 2, muscle strength for flexion and extension at the ankle and knee and Neuropathy Impairment Score (NIS) are included. In model 3, muscle strength measurements at the ankle and knee are combined. In model 4, NIS and MVD are combined. All data are shown as odds ratios (OR) and 95\% confidence intervals (CI 95\%).

\begin{tabular}{|c|c|c|}
\hline & OR $(95 \%$ CI) & $\mathbf{P}$ \\
\hline \multicolumn{3}{|l|}{ Model 1} \\
\hline $\begin{array}{l}\text { a) Ankle dorsal flexion } \\
\text { MVD }\end{array}$ & $\begin{array}{l}1.06(1.02-1.09) \\
4.15(1.40-12.27)\end{array}$ & $\begin{array}{l}<0.005 \\
<0.05\end{array}$ \\
\hline $\begin{array}{l}\text { b) Ankle plantar flexion } \\
\text { MVD }\end{array}$ & $\begin{array}{c}1.04(1.01-1.07) \\
4.96(1.74-14.18)\end{array}$ & $\begin{array}{l}<0.05 \\
<0.05\end{array}$ \\
\hline $\begin{array}{l}\text { c) Knee extension } \\
\text { MVD }\end{array}$ & $\begin{array}{c}1.09(1.03-1.15) \\
5.21(1.61-16.91)\end{array}$ & $\begin{array}{l}<0.001 \\
<0.05\end{array}$ \\
\hline $\begin{array}{l}\text { d) Knee flexion } \\
\text { MVD }\end{array}$ & $\begin{array}{c}1.06(1.02-1.10) \\
8.03(2.43-26.55)\end{array}$ & $\begin{array}{l}<0.005 \\
<0.005\end{array}$ \\
\hline \multicolumn{3}{|l|}{ Model 2} \\
\hline $\begin{array}{l}\text { a) Ankle dorsal flexion } \\
\text { NIS }\end{array}$ & $\begin{array}{l}1.03(0.99-1.07) \\
1.12(1.05-1.19)\end{array}$ & $\begin{aligned} & 0.08 \\
< & 0.001\end{aligned}$ \\
\hline $\begin{array}{l}\text { b) Ankle plantar flexion } \\
\text { NIS }\end{array}$ & $\begin{array}{l}1.02(0.98-1.05) \\
1.13(1.07-1.21)\end{array}$ & $\begin{aligned} & 0.4 \\
< & 0.001\end{aligned}$ \\
\hline $\begin{array}{l}\text { c) Knee extension } \\
\text { NIS }\end{array}$ & $\begin{array}{l}1.04(0.98-1.09) \\
1.12(1.05-1.20)\end{array}$ & $\begin{aligned} & 0.18 \\
< & 0.005\end{aligned}$ \\
\hline $\begin{array}{l}\text { d) Knee flexion } \\
\text { NIS }\end{array}$ & $\begin{array}{l}1.03(0.99-1.07) \\
1.14(1.07-1.21)\end{array}$ & $\begin{aligned} & 0.17 \\
< & 0.001\end{aligned}$ \\
\hline \multicolumn{3}{|l|}{ Model 3} \\
\hline $\begin{array}{l}\text { a) Ankle dorsal flexion } \\
\text { Ankle plantar flexion }\end{array}$ & $\begin{array}{l}1.05(1.02-1.08) \\
1.02(0.99-1.06)\end{array}$ & $\begin{array}{c}<0.005 \\
0.13\end{array}$ \\
\hline $\begin{array}{l}\text { b) Knee extension } \\
\text { Knee flexion }\end{array}$ & $\begin{array}{l}1.08(1.02-1.15) \\
1.02(0.96-1.05)\end{array}$ & $\begin{array}{c}<0.05 \\
0.77\end{array}$ \\
\hline $\begin{array}{l}\text { c) Ankle dorsal flexion } \\
\text { Knee extension }\end{array}$ & $\begin{array}{l}1.06(1.02-1.1) \\
1.05(1.0-1.11\end{array}$ & $\begin{array}{c}<0.01 \\
0.06\end{array}$ \\
\hline \multicolumn{3}{|l|}{ Model 4} \\
\hline $\begin{array}{l}\text { NIS } \\
\text { MVD }\end{array}$ & $\begin{array}{c}1.14(1.08-1.21) \\
3.47(1.07-11.30)\end{array}$ & $\begin{array}{l}<0.001 \\
<0.05\end{array}$ \\
\hline
\end{tabular}

Further, we have illustrated the effect of muscle weakness on DFU development over time, strengthening the hypothesis of an association between alterations in gait due to loss of muscle strength and foot ulcer formation, possibly as a result of increased plantar pressure.

An association to DFU development was also found for MVD and NIS in both uni- and multivariate analysis, lending support to the multifactorial pathology presumed to underlie the development of foot ulcers in diabetes.

Several studies have described gait alterations in diabetic patients with DPN. Late firing of the anterior tibial muscle has been found to result in forefoot slap and, resultantly, increased plantar pressure, which contributes to development of DFU $[15,24]$. In our study, reduced muscle strength for dorsal flexion at the ankle showed a close correlation to foot ulceration, thus our results support these findings as the anterior tibial muscle contributes to ankle dorsiflexion. A correlation between DFU and muscle strength for ankle dorsal flexion, toe extension, and finger abduction has been reported, however, muscle strength was evaluated manually [18]. As our muscle strength measurements were acquired using a standardized quantitative technique with a low coefficient of variation, and included ankle extension and muscle strength at the knee in addition to ankle dorsal flexion, the results bring substantial support to the association between muscle weakness in DPN and DFU occurrence. Patients with DPN experience atrophy of foot muscles, and a relationship between prior DFU and weakness of intrinsic as well as extrinsic foot muscles calculated by a semi quantitative scoring system has been reported [25]. As DPN is distributed in a length-dependent manner, an evaluation of the relationship between foot muscle weakness and DFU would have added valuable information, however these data were not obtained in the initial studies as dynamometric strength measurements of small foot muscles are not easily performed. Due to the retrospective nature of our study, important risk factors for foot ulceration associated to increased plantar pressure such as foot deformities and callus formation could not be adequately acquired.

The severity of DPN is a well-known risk factor for diabetic foot ulceration, a finding also supported in our study. When adjusting for the severity of DPN, expressed as NIS, in the multivariate analysis, strength of the ankle dorsal flexors no longer showed an association to foot ulceration. However, a trend towards a relationship between the variable and DFU development remained. This observation is not surprising since reduced muscle strength is caused by motor neuropathy, which occurs concomitantly with sensory neuropathy in DPN [26].

MVD was strongly associated to foot ulceration, a finding supported by other groups $[3,5,27]$. However, the influence of the association found in our study is most probably overestimated, as data on MVD were recorded consecutively throughout the follow-up period, and are therefore, unlike all strength measurements, NIS, and VPT, not baseline data.

VPT showed no relationship to DFU development when applying univariate analysis, although sensory neuropathy is a well-known risk factor for the development of DFU and increased thresholds for vibration occur in early stages of DPN [18]. In our analyses many 
patients presented with VPT exceeding the arbitrary unit 25 JND (Just Noticeable Difference), thus the lack of correlation may be due to a ceiling effect.

Increased physical activity has been suggested to play a role in DFU development due to repetitive mechanical stress of the plantar region of the foot. However, no increase in foot ulcer occurrence was observed among neuropathic diabetic patients enrolled in a twelvemonth muscle-strengthening program, and further, another study suggested an overall lower activity in diabetic patients who developed DFU compared to those without this complication $[28,29]$. Thus, there are no convincing data to suggest that physical activity increases the risk of foot ulcer formation. It has been reported that specific training of the lower extremities in diabetic patients diagnosed with sensory neuropathy improve gait speed, balance, muscle strength and joint mobility [30]. As reduced muscle strength seems to be implicated in foot ulceration in diabetes, strengthening of lower extremity muscles may contribute to preservation of normal gait, and thereby reduce the risk of developing foot ulcers. Also, a randomized controlled trial evaluating the effect of leg muscle strengthening and gait exercises in neuropathic diabetic patients, reported a more expedient distribution of plantar pressure and gait execution [31]. However, as intentionto-treat analyses showed improvement and the intervention only administered for twelve weeks, exercises directed at enhancing lower extremity function may still play a role in DFU prevention. Based on the aforementioned study and our findings, a study evaluating the effect of strengthening of lower extremity muscles on DFU formation is highly relevant, however both the intervention and follow-up period must be of sufficient length as the mechanisms leading to DFU exert their detrimental impact over years.

In conclusion, we found a temporal relationship between reduced muscle strength at the knee and ankle and diabetic foot ulcer development. Notably reduced muscle strength for dorsal flexion at the ankle seemed to have a close relationship to foot ulceration, lending support to previous findings evaluating gait biomechanics in DPN and the proposed relationship between increased plantar pressure and DFU development. As motor dysfunction in DPN is a contributing factor in the multifactorial pathway involved in foot ulceration, it would be relevant to include quantitative strength measurements and gait analyses in large scaled prospective studies investigating the aetiology behind this feared complication to diabetes mellitus.

Authorship: A.B.P wrote the manuscript, took part in designing the study, and collected and analysed data. L.H. contributed to study design, data collection and analyses, and reviewed the manuscript. H.A. contributed to study conception and design, analyses, and reviewed/ edited the manuscript. N.E. contributed to design, data collection, discussion, and reviewed the manuscript. C.S.A. contributed to study conception and design, researched data, conducted statistical analyses, and reviewed and edited the manuscript. All authors approved the final version of the manuscript. A.B.P. is guarantor of this work.

\section{Acknowledgment}

Results of the study were presented in a shortened form at the 21st Annual Meeting of the Diabetic Neuropathy Study Group of the EASD, held in Porto, Portugal, 8-11 September 2011.

\section{References}

1. American Diabetes Association (2008) Economic costs of diabetes in the U.S. In 2007. Diabetes Care 31: 596-615. [crossref]

2. Vileikyte L (2001) Diabetic foot ulcers: a quality of life issue. Diabetes/metabolism research and reviews 17: 246-249.

3. Abbott CA, Carrington AL, Ashe H, Bath S, Every LC, et al. (2002) The NorthWest Diabetes Foot Care Study: incidence of, and risk factors for, new diabetic foot ulceration in a community-based patient cohort. Diabetic medicine : a journal of the British Diabetic Association 19: 377-84.

4. Adler AI, Boyko EJ, Ahroni JH, Stensel V, Forsberg RC, et al. (1997) Risk factors for diabetic peripheral sensory neuropathy. Results of the Seattle Prospective Diabetic Foot Study. Diabetes care 20: 1162-1167.

5. Boyko EJ, Ahroni JH, Stensel V, Forsberg RC, Davignon DR, et al. (1999) A prospective study of risk factors for diabetic foot ulcer. The Seattle Diabetic Foot Study. Diabetes Care 22: 1036-1042. [crossref]

6. McNeely MJ, Boyko EJ, Ahroni JH, Stensel VL, Reiber GE, et al. (1995) The independent contributions of diabetic neuropathy and vasculopathy in foot ulceration. How great are the risks? Diabetes Care 18: 216-219. [crossref]

7. Shun CT, Chang YC, Wu HP, Hsieh SC, Lin WM, et al. (2004) Skin denervation in type 2 diabetes: correlations with diabetic duration and functional impairments. Brain 127: 1593-1605.

8. Andersen H, Gjerstad MD, Jakobsen J (2004) Atrophy of foot muscles: a measure of diabetic neuropathy. Diabetes Care 27: 2382-2385. [crossref]

9. Andersen H, Nielsen S, Mogensen CE, Jakobsen J (2004) Muscle strength in type 2 diabetes. Diabetes 53: 1543-1548. [crossref]

10. Andreassen CS, Jakobsen J, Andersen H (2006) Muscle weakness: a progressive late complication in diabetic distal symmetric polyneuropathy. Diabetes 55: 806812. [crossref]

11. Andreassen CS, Jakobsen J, Ringgaard S, Ejskjaer N, Andersen H (2009) Accelerated atrophy of lower leg and foot muscles--a follow-up study of long-term diabetic polyneuropathy using magnetic resonance imaging (MRI). Diabetologia 52: 11821191. [crossref]

12. Cheuy VA, Hastings MK, Commean PK, Ward SR, Mueller MJ (2013) Intrinsic foot muscle deterioration is associated with metatarsophalangeal joint angle in people with diabetes and neuropathy. Clinical biomechanics (Bristol, Avon) 28: 1055-1060.

13. Raspovic A (2013) Gait characteristics of people with diabetes-related peripheral neuropathy, with and without a history of ulceration. Gait \& posture 38: 723-728.

14. van Schie CH (2005) A review of the biomechanics of the diabetic foot. Int J Low Extrem Wounds 4: 160-170. [crossref]

15. Abboud RJ, Rowley DI, Newton RW (2000) Lower limb muscle dysfunction may contribute to foot ulceration in diabetic patients. Clinical biomechanics (Bristol, Avon) 15: 37-45.

16. Frykberg RG, Lavery LA, Pham H, Harvey C, Harkless L, et al. (1998) Role of neuropathy and high foot pressures in diabetic foot ulceration. Diabetes Care 21: 1714-1719. [crossref]

17. Stess RM, Jensen SR, Mirmiran R (1997) The role of dynamic plantar pressures in diabetic foot ulcers. Diabetes Care 20: 855-858. [crossref]

18. Abbott CA, Vileikyte L, Williamson S, Carrington AL, Boulton AJ (1998) Multicenter study of the incidence of and predictive risk factors for diabetic neuropathic foot ulceration. Diabetes care 21: 1071-1075.

19. Andersen H, Jakobsen J (1997) A comparative study of isokinetic dynamometry and manual muscle testing of ankle dorsal and plantar flexors and knee extensors and flexors. European neurology 37: 239-242.

20. Andersen H (1996) Reliability of isokinetic measurements of ankle dorsal and plantar flexors in normal subjects and in patients with peripheral neuropathy. Arch Phys Med Rehabil 77: 265-268. [crossref]

21. Andersen H, Poulsen PL, Mogensen CE, Jakobsen J (1996) Isokinetic muscle strength in long-term IDDM patients in relation to diabetic complications. Diabetes 45: 440-445.

22. Dyck PJ, O’Brien PC, Kosanke JL, Gillen DA, Karnes JL (1993) A 4, 2, and 1 stepping algorithm for quick and accurate estimation of cutaneous sensation threshold. Neurology 43: 1508-1512. [crossref]

23. Peduzzi P, Concato J, Kemper E, Holford TR, Feinstein AR (1996) A simulation study of the number of events per variable in logistic regression analysis. J Clin Epidemiol 49: 1373-1379. [crossref]

24. Shaw JE, van Schie CH, Carrington AL, Abbott CA, Boulton AJ (1998) An analysis of dynamic forces transmitted through the foot in diabetic neuropathy. Diabetes care 21: 1955-1959.

25. van Schie CH, Vermigli C, Carrington AL, Boulton A (2004) Muscle weakness and foot deformities in diabetes: relationship to neuropathy and foot ulceration in caucasian diabetic men. Diabetes care 27: 1668-1673. 
26. Dyck PJ, Albers JW, Andersen H, Arezzo JC, Biessels GJ, et al. (2011) Diabetic polyneuropathies: update on research definition, diagnostic criteria and estimation of severity. Diabetes/metabolism research and reviews. 27: 620-628.

27. Monteiro-Soares M, Dinis-Ribeiro M (2010) External validation and optimisation of a model for predicting foot ulcers in patients with diabetes. Diabetologia 53: 1525-1533. [crossref]

28. Lemaster JW, Mueller MJ, Reiber GE, Mehr DR, Madsen RW, et al. (2008) Effect of weight-bearing activity on foot ulcer incidence in people with diabetic peripheral neuropathy: feet first randomized controlled trial. Physical Therapy 88: 1385-1398.

29. Armstrong DG, Lavery LA, Holtz-Neiderer K, Mohler MJ, Wendel CS, et al. (2004) Variability in activity may precede diabetic foot ulceration. Diabetes Care 27: 1980-1984. [crossref]
30. Allet L, Armand S, de Bie RA, Golay A, Monnin D, et al. (2010) The gait and balance of patients with diabetes can be improved: a randomised controlled trial. Diabetologia 53: 458-466. [crossref]

31. Sartor CD, Hasue RH, Cacciari LP, Butugan MK, Watari R, et al. (2014) Effects of strengthening, stretching and functional training on foot function in patients with diabetic neuropathy: results of a randomized controlled trial. BMC musculoskeletal disorders 15: 137-2474-15-137.

Citation:

Bruno Pedersen AS, Junggreen Have LW, Andersen H, Ejskjaer N, Swan CZ (2019) Risk of Foot Ulcer Development in Diabetic Patients - Relation to Isokinetic Muscle Strength, Sensory Function, and Clinical Findings. Endocrinol Diabetes Metab J Volume 3(3): 1-6. 